\title{
Candidemia em hospital terciário do nordeste do Brasil
}

\author{
Candidemia in a tertiary hospital in northeastern Brazil
}

\author{
Sylvia Lemos Hinrichsen ${ }^{1}$, Érika Falcão ${ }^{2}$, Tatiana Aguiar Santos Vilella ${ }^{1}$, \\ Arnaldo Lopes Colombo ${ }^{3}$, Márcio Nucci ${ }^{4}$, Líbia Moura ${ }^{1}$, Leandro Rêgo ${ }^{5}$, \\ Conceição Lira ${ }^{1}$ e Luciano Almeida ${ }^{2}$
}

\begin{abstract}
RESUMO
Realizou-se um estudo observacional, prospectivo, de base laboratorial, para investigar a incidência de candidemia, distribuição de espécies e condições clínicas entre setembro 2003 e março 2004, em um hospital privado terciário em Recife, Nordeste do Brasil. Um caso de candidemia foi definido como isolamento de Candida spp de hemocultura. A taxa de incidência foi calculada por 1.000 admissões. Um total de 5.532 pacientes foram admitidos no hospital durante o período de estudo, e 1.745 culturas de sangue foram processadas. Foram observados 21 episódios de candidemia em 18 pacientes. A taxa de incidência de candidemia foi de 3,9 episódios por 1.000 admissões. Espécies não-albicans representaram mais de 50\% dos casos, predominando Candida parapsilosis (33\%) e Candida tropicalis (24\%). Onze (61\%) pacientes morreram. A incidência de candidemia foi mais alta que aquela observada em estudo multicêntrico brasileiro. Candidemia foi predominantemente causada por espécies não-albicans.
\end{abstract}

Palavras-chaves: Candidemia. Espécies de Candida. Candida não-albicans. Incidência.

\begin{abstract}
We conducted a prospective, observational, laboratory-based study on candidemia to investigate the incidence of candidemia, species distribution and clinical conditions between September 2003 and March 2004 in a private tertiary hospital in Recife, northeastern Brazil. Cases of candidemia were defined as occurrences of isolation of Candida spp from blood cultures. The incidence rate was calculated per 1,000 admissions. A total of 5,532 patients were admitted to the hospital during the study period, and 1,745 blood cultures were processed. Twenty-one episodes of candidemia were observed in 18 patients. The incidence rate of candidemia was 3.9 episodes per 1,000 admissions. Non-albicans species accounted for more than 50\% of the cases, and Candida parapsilosis (33\%) and Candida tropicalis (24\%) predominated. Eleven (61\%) patients died. The incidence of candidemia was higher than that observed in a Brazilian multicenter study. Candidemia was caused predominantly by non-albicans species.
\end{abstract}

Key-words: Candidemia. Candida species. Candida non-albicans. Incidence.

A freqüência de infecções hematogênicas por Candida (candidemia) tem aumentado consideravelmente, especialmente em unidades de terapia intensiva e ou de assistência a pacientes $\operatorname{críticos}^{234781415172024}$. 0 aumento na freqüência de candidemia tem sido observado particularmente entre pacientes em uso de antibióticos, terapia imunossupressora, nutrição parenteral, e em pacientes expostos a múltiplos procedimentos invasivos ${ }^{11} 24$.

Espécies do gênero Candida, em particular Candida albicans, têm emergido como importantes patógenos nosocomiais, estando associadas a quase $80 \%$ de todas as infecções fúngicas nosocomiais, representando a maior causa de fungemia ${ }^{24}$. Candidemia é a quarta causa mais comum de infecção na corrente sangüínea em hospitais terciários, e sua ocorrência tem sido associada à longa permanência hospitalar e alta mortalidade ${ }^{101821}$.
No Brasil, as principais espécies causadoras de candidemia são Candida albicans, Candida parapsilosis e Candida tropicalis $^{79}$. Diferente dos Estados Unidos da América (EUA), onde a emergência de espécies não-albicans parece associada à pressão seletiva do uso de fluconazol, no Brasil as espécies não-albicans mais prevalentes são sensíveis a este fármaco ${ }^{19}$.

Considerando a grande variabilidade na epidemiologia de candidemia observada em diferentes serviços médicos e a importância do conhecimento do perfil microbiológico destas infecções para a definição de medidas de prevenção, controle e tratamento, o presente estudo teve como objetivo determinar a incidência de candidemia nosocomial, as espécies prevalentes de Candida e as populações susceptíveis.

\footnotetext{
1. Núcleo de Ensino, Pesquisa e Assistência em Infectologia, Universidade Federal de Pernambuco, Recife, PE. 2. Departamento de Microbiologia, Cerpe Diagnósticos, Recife, PE. 3. Disciplina de Doenças Infecciosas e Parasitárias, Universidade Federal de São Paulo, São Paulo, SP. 4. Laboratório de Micologia, Hospital Universitário Clementino Fraga Filho, Universidade Federal do Rio de Janeiro, Rio de Janeiro, RJ. 5. Departamento de Estatística, Universidade Federal de Pernambuco, Recife, PE. Apoio financeiro: CNPq/PIBIC/UFPE. Endereço para correspondência: Dra. Sylvia Lemos Hinrichsen. Rua Jornalista Guerra de Holanda 158/2601, Casa Forte, 52061-010 Recife PE. Tel: $55813268-9905$

e-mail: sylviahinrichsen@hotmail.com Recebido para publicação em 22/11/2007 Aceito em 26/07/2008
} 


\section{MATERIAL E MÉTODOS}

Realizou-se um estudo prospectivo, de base laboratorial, num período de seis meses ( $1^{\circ}$ de setembro de 2003 a $1^{\circ}$ de março de 2004), em um hospital terciário geral, privado, de alta complexidade, localizado em Recife, com 217 leitos, incluindo a maioria das especialidades da clínica médica e cirúrgica, unidade de terapia intensiva (UTI) de adulto (clínica e cirúrgica com 15 leitos), UTI neonatal (12 leitos), UTI pediátrica (quatro leitos), unidade coronária (12 leitos); emergência de adulto/pediátrica/ cardiológica e serviço de ginecologia e obstetrícia. Os pacientes eram procedentes da clínica privada/ convênios, referenciados de outros hospitais e ou da comunidade.

Os pacientes estudados foram identificados a partir da vigilância de resultados de hemoculturas gerados pelo laboratório de microbiologia da instituição. Considerou-se um caso incidente de candidemia o primeiro isolamento de qualquer espécie de Candida de uma cultura de sangue de um paciente com sinais e sintomas de sepse. Um episódio de candidemia referiu-se a uma candidemia incidente em período de 30 dias após a data da coleta da primeira hemocultura positiva. Se uma outra hemocultura positiva ocorresse durante estes 30 dias, seja pela mesma espécie ou por outra espécie diferente de Candida, esta hemocultura era registrada como parte do mesmo episódio de candidemia. Entretanto, se um paciente apresentasse outra hemocultura positiva $>30$ dias depois da cultura incidente (primeira hemocultura), esta era contada como novo episódio, e uma nova ficha de candidemia era preenchida.

Durante o período do estudo, foram internados 5.532 pacientes e processadas 1.745 culturas de sangue pelo sistema comercial BactAlert (Biomérieux AS, France). As hemoculturas positivas foram cultivadas em meio Sabouraud-dextrose, e as colônias isoladas foram processadas pelo sistema automatizado Vitek (Biomérieux AS, France) para a identificação das espécies de Candida.

Utilizando-se uma ficha clínica padrão, foram documentados os dados clínicos e epidemiológicos dos pacientes com candidemia através da revisão de prontuários médicos, sendo as informações armazenadas em banco de dados, utilizando os programas Microsoft Excel 2000 versão 9.0.2812 e Epi Info versão 3.3.2.
As variáveis estudadas foram: idade, gênero, doenças de base, condições clínicas, presença de cateter venoso central, cirurgia prévia, uso de nutrição parenteral, corticoterapia, presença e duração de neutropenia e antifúngicos prescritos. Foram consideradas como condições de risco para candidemia aquelas presentes até 30 dias antes da ocorrência da infecção. As taxas de incidência foram calculadas por 1.000 admissões hospitalares. 0 seguimento dos casos a partir das informações clínicas sobre a evolução dos pacientes foram coletadas até o fim da hospitalização (alta hospitalar) ou até o óbito. Todos os dados foram analisados através de estatística descritiva. 0 estudo obteve a aprovação do comitê de ética em pesquisa da Universidade Federal de Pernambuco ( $\left.{ }^{0} 1.263 / 2003\right)$.

\section{RESULTADOS}

Foram observados 21 episódios de candidemia em 18 pacientes; três pacientes tiveram um segundo episódio de candidemia. Candida parapsilosis $\left(\mathrm{n}^{\circ}=7\right)$ e Candida albicans $\left(\mathrm{n}^{0}=6\right)$ foram as espécies mais encontradas (Tabela 1).

Dos 21 episódios, treze (62\%) ocorreram em homens. A idade dos pacientes variou de um mês a 89 anos (mediana de 59 anos). Do total de episódios, sete (33\%) ocorreram em pacientes com idade $<20$ anos, quatro (19\%) em pacientes entre 20 e 60 anos e dez (48\%) em pacientes $>60$ anos. Os episódios de candidemia ocorreram predominantemente em pacientes internados na unidade de terapia intensiva $\left(n^{\circ}=13\right)$, dos quais oito do tipo geral (Tabela 2).

Nenhum dos pacientes tinha sido transferido de outro setor de internação nas 48 horas anteriores à candidemia. Seis (29\%) episódios ocorreram em pacientes que tiveram bacteremia nos 14 dias antes ou no dia da candidemia, e em três (14\%) Candida spp foi isolada de outro sítio (urina).

A doença de base mais predominante foi a pulmonar (48\%), seguida pela neurológica (33\%), cardíaca (29\%) e hepática (29\%) (Tabela 3).

Entre as condições associadas mostradas na Tabela 4 , as mais frequientes foram uso de antibióticos (90\%), internamento na unidade de terapia intensiva $(62 \%)$, presença de cateter venoso central (57\%) e uso de corticoesteróide (52\%).

Tabela 1 - Distribuição das espécies de Candida spp causadoras de 21 episódios de candidemia. Recife, 2003-2004.

\begin{tabular}{lccc}
\hline Espécies & Número & Porcentagem & $\begin{array}{c}\text { Incidência por } \\
1.000 \text { admissões }\end{array}$ \\
\hline Candida albicans & 6 & 29,0 & 1,1 \\
Candida não-albicans & & & \\
$\quad$ Candida parapsilosis & 7 & 33,0 & 1,3 \\
$\quad$ Candida tropicalis & 5 & 24,0 & 0,9 \\
$\quad$ Candida glabrata & 2 & 9,0 & 0,4 \\
Candida guilliermondii & 1 & 5,0 & 0,2 \\
\hline Total & 21 & 100,0 & 3,9 \\
\hline
\end{tabular}


Tabela 2 - Características dos 21 episódios de candidemia ocorridos em 18 pacientes. Recife, 2003-2004.

\begin{tabular}{|c|c|c|c|c|c|c|c|}
\hline $\begin{array}{l}\text { Episódio/ } \\
\text { Paciente }\end{array}$ & Sexo & $\begin{array}{l}\text { Idade } \\
\text { meses/anos }\end{array}$ & $\begin{array}{l}\text { Unidade de } \\
\text { internação }\end{array}$ & $\begin{array}{l}\text { Espécie } \\
\text { Candida }\end{array}$ & $\begin{array}{l}\text { Óbito em } \\
30 \text { dias }\end{array}$ & Antifúngico prévio & Tratamento \\
\hline $1-\mathrm{A}$ & $\mathrm{F}$ & 47 & UTI geral & glabrata & $\operatorname{sim}$ & fluconazol & $\begin{array}{l}\text { anfotericina B desoxicolato } \rightarrow \\
\text { caspofungina }\end{array}$ \\
\hline $2-B$ & $\mathrm{~F}$ & 54 & UTI geral & albicans & não & não & não \\
\hline $3-\mathrm{C}$ & $\mathrm{M}$ & 86 & Clínica médica & tropicalis & $\operatorname{sim}$ & não & anfotericina B lipossomal \\
\hline $4-D$ & $\mathrm{~F}$ & 89 & UTI geral & glabrata & não & não & anfotericina B lipossomal \\
\hline $5-\mathrm{D}$ & $\mathrm{F}$ & 89 & Clínica médica & parapsilosis & $\operatorname{sim}$ & não & anfotericina a B lipossomal \\
\hline $6-E$ & M & 9 & Pediatria & albicans & não & não & não \\
\hline$\overline{7-F}$ & $\mathrm{~F}$ & 59 & Clínica médica & guilliermondii & $\operatorname{sim}$ & fluconazol & fluconazol \\
\hline $8-G$ & M & 84 & UTI geral & tropicalis & $\operatorname{sim}$ & não & não \\
\hline $9-\mathrm{H}$ & M & 1 mês & UTI neonatal & parapsilosis & $\operatorname{sim}$ & não & anfotericina B lipossomal \\
\hline $10-I$ & $\mathrm{~F}$ & 7 & UTI pediátrica & parapsilosis & não & anfotericina B lipossomal & anfotericina B lipossomal \\
\hline $11-\mathrm{I}$ & $\mathrm{F}$ & 7 & UTI pediátrica & parapsilosis & $\operatorname{sim}$ & fluconazol & fluconazol \\
\hline $12-\mathrm{J}$ & $\mathrm{F}$ & 71 & UTI geral & tropicalis & $\operatorname{sim}$ & anfontericina $\mathrm{B}$ desoxicolato & não \\
\hline $13-\mathrm{K}$ & $\mathrm{M}$ & 4 & UTI pediátrica & parapsilosis & não & não & não \\
\hline $14-K$ & M & 4 & UTI pediátrica & parapsilosis & não & não & não \\
\hline $15-\mathrm{L}$ & $\mathrm{M}$ & 76 & UTI geral & albicans & $\operatorname{sim}$ & caspofungina & anfotericina B lipossomal \\
\hline $16-M$ & $\mathrm{M}$ & 88 & UTI geral & tropicalis & $\operatorname{sim}$ & não & $\begin{array}{l}\text { anfotericina B desoxicolato } \rightarrow \text { seguida } \\
\text { de anfotericina B lipossomal }\end{array}$ \\
\hline $17-\mathrm{N}$ & M & 78 & Clínica médica & albicans & não & não & fluconazol \\
\hline $18-0$ & $\mathrm{M}$ & 83 & UTI geral & albicans & $\operatorname{sim}$ & anfontericina B lipossomal & anfotericina B lipossomal \\
\hline $19-\mathrm{P}$ & M & 65 & UTI cardíaca & tropicalis & não & não & fluconazol \\
\hline $20-Q$ & $\mathrm{M}$ & 29 & Clínica médica & albicans & não & não & não \\
\hline $21-\mathrm{R}$ & $\mathrm{M}$ & 7 meses & UTI neonatal & parapsilosis & não & fluconazol & fluconazol \\
\hline
\end{tabular}

UTI: unidade de terapia intensiva, F: feminino, M: masculino

Tabela 3 - Doença de base dos 21 episódios de candidemia ocorridos em 18 pacientes. Recife, 2003-2004.

\begin{tabular}{lcc}
\hline Doença de base & Número* $^{*}$ & Porcentagem \\
\hline Doença pulmonar & 10 & 48,0 \\
Doença neurológica & 7 & 33,0 \\
Doença cardíaca & 6 & 29,0 \\
Doença hepática & 6 & 29,0 \\
Insuficiência renal & 5 & 24,0 \\
Neoplasias & 4 & 19,0 \\
Doença auto-imune & 1 & 4,8 \\
\hline
\end{tabular}

*número de vezes que aparecem.

A taxa de mortalidade foi de $61 \%\left(n^{0}=11\right)$, apesar da maioria (62\%) ter recebido tratamento antifúngico. No grupo de óbitos houve predomínio dos adultos, dos quais sete (78\%) tinham idade $>60$ anos e oito (88\%) estavam internados em unidade de terapia intensiva. Dos três pacientes que apresentaram um segundo episódio de candidemia, durante a hospitalização, apenas um sobreviveu após período de 30 dias do diagnóstico e recebeu alta hospitalar.

Tabela 4 - Condições associadas nos pacientes com candidemia no bospital terciário em Recife, 2003-2004.

\begin{tabular}{lcc}
\hline Condições associadas & Número* $^{*}$ & Porcentagem \\
\hline Antibióticos & 19 & 90,0 \\
Internação na unidade de terapia itensiva & 13 & 62,0 \\
Cateter venoso central & 12 & 57,0 \\
Corticoesteróide & 11 & 52,0 \\
Nutrição parenteral total & 5 & 24,0 \\
Bloqueador $\mathrm{H}_{2}$ & 3 & 14,0 \\
Imunossupressor & 1 & 4,8 \\
\hline
\end{tabular}

*número de vezes que aparecem.

\section{DISCUSSÃO}

No Brasil, a incidência de candidemia foi analisada prospectivamente em 11 centros hospitalares públicos das regiões Sul, Sudeste e do Distrito Federal ${ }^{9}$. Observou-se uma taxa de 2,4 casos por 1.000 admissões hospitalares, enquanto estudos em hospitais terciários dos EUA e da Europa observaram taxas muito mais baixas, em geral menores que um episódio de candidemia por 1.000 admissões hospitalares ${ }^{923}$. A incidência encontrada no presente trabalho, de 3,9 por 1.000 admissões hospitalares, esteve acima das referidas em outros países e em centros brasileiros ${ }^{291123}$. As razões para esta alta taxa de candidemia em hospitais brasileiros, e uma taxa ainda maior nessa casuística, não são claras. Entretanto nos dados brasileiros, observou-se que a taxa de infecções na corrente sangüínea por bactérias também era muito mais alta que aquela relatada em estudos no hemisfério norte. Estes dados apontam para um problema comum à bactérias e fungos causando infecção na corrente sangüínea e não só candidemia. É possível que estas diferenças ocorram por diversos padrões de atenção a doentes graves, tais como número de profissionais de saúde por paciente, qualificação dos profissionais de saúde, reutilização de material descartável, utilização de sistema aberto de infusão venosa, e adesão a práticas padrão de controle de infecção hospitalar. Outros estudos são necessários para melhor esclarecer esta questão.

Acredita-se que a maioria dos casos de candidemia seja adquirida por via endógena, pela translocação de Candida através do trato gastrintestinal ${ }^{1}$. Entretanto, infecções hematogênicas por Candida spp também podem ser adquiridas por via exógena, 
através do contato das mãos de profissionais de saúde com pacientes portadores de cateteres vasculares centrais, implante de próteses contaminadas, bem como pela administração parenteral de soluções contaminadas ${ }^{6}$.

A predominância de Candida parapsilosis entre as crianças observada neste trabalho é similar a de outros estudos ${ }^{811} 1617$. Candia parapsilosis é considerado agente de infecções exógenas por ser capaz de colonizar a pele, principalmente as mãos de profissionais da saúde, assim como as soluções glicosiladas de uso hospitalar e cateter venoso central. Sua detecção é particularmente associada à nutrição parenteral total. A ocorrência de Candida parapsilosis é maior em crianças, principalmente em prematuros internados em unidades de terapia intensiva ${ }^{82}$.

São condições associadas para o desenvolvimento de candidemia em unidades de terapia intensiva a gravidade da doença de base, uso de esteróides, múltiplas transfusões, nutrição parenteral, cirurgias de grande porte, hemodiálise, cateter venoso central, pancreatite, além do tempo de hospitalização e uso de antibióticos de amplo espectro ${ }^{1124}$. No presente estudo, observou-se que oito (38\%) episódios de candidemia ocorreram em pacientes que estavam internados em unidade de terapia intensiva geral e quatro (19\%) em UT'I pediátrica, dados esses que estão de acordo com outros relatos, que mostram uma maior prevalência de casos de candidemia entre pacientes críticos internados em centros de tratamento intensivo e com vários fatores de risco ${ }^{9}{ }^{19}$.

Nos últimos anos vem aumentando o número de infecções invasivas causadas por espécies de Candida não-albicans ${ }^{91323}$. Sabese que na América Latina há predomínio de Candida tropicalis e Candida parapsilosis entre as espécies não-albicans causadoras de candidemia, sendo pouco freqüente a ocorrência de fungemias por Candida glabrata 7 . Já nos EUA e em muitos países da Europa, predomina Candida glabrata entre as não-albicans ${ }^{13}$.

Em 712 episódios de candidemia de hospitais públicos do Brasil, observou-se predomínio da Candida albicans (41\%) seguida de Candida tropicalis (21\%) e Candida parapsilosis $(20 \%)^{7}$. Nessa casuística, as espécies não-albicans foram mais frequientes que Candida albicans, e destas, Candida parapsilosis $\left(\mathrm{n}^{0}=7\right)$ foi a mais encontrada, seguida da Candida tropicalis $\left(n^{0}=5\right)$. Apesar dos números pequenos, e do caráter unicêntrico deste estudo, os dados encontrados estão de acordo com 0 estudo epidemiológico brasileiro que demonstrou que Candida albicans, Candida parapsilosis e Candida tropicalis causam a maioria das candidemias ${ }^{9}$. Embora tenha sido observada uma maior proporção de candidemia por Candida glabrata (9\%) comparado com os dados brasileiros ${ }^{9}$, o número pequeno da candidemias não permite uma conclusão definitiva. A epidemiologia de candidemia em centros do Nordeste brasileiro não é ainda muito conhecida5 .

A identificação das espécies de Candida também facilita a abordagem terapêutica a ser instituída, uma vez que algumas espécies não menos susceptíveis a fluconazol, como Candida krusei e Candida glabrata, e Candida lusitaniae podem ser resistentes a anfotericina $\mathrm{B}^{19}$. Pacientes com maior risco para o desenvolvimento de fungemias por cepas resistentes a fluconazol são representados por aqueles previamente expostos a este medicamento, portadores de doenças malignas, neutropênicos, expostos a transplantes de órgãos, portadores da síndrome da imunodeficiência humana (AIDS/SIDA), bem com antecedentes de internação prévia ${ }^{19}$. Os achados deste trabalho, embora limitados pelo pequeno tamanho da amostra, são importantes, uma vez que os estudos brasileiros de candidemia, publicados na literatura, foram realizados nas regiões Sul e Sudeste, e em hospitais públicos terciários, sendo escassas as informações sobre a epidemiologia de candidemia em hospitais privados brasileiros ${ }^{711}$.

Sete dos 14 pacientes tratados receberam anfotericina B lipossomal. Esta droga é uma das opções terapêuticas de candidemia, como relatado em estudo randomizado, recentemente publicado ${ }^{12}$. Estes dados não refletem a realidade dos principais hospitais públicos brasileiros, onde anfotericina $\mathrm{B}$ desoxicolato e fluconazol respondem pela maioria dos tratamentos primários de candidemia?

A principal limitação deste estudo é o pequeno número de episódios de candidemia. Entretanto, estes dados mostram a incidência de candidemia neste hospital, além de apresentar, ainda que de forma limitada, dados epidemiológicos de candidemia em uma instituição privada localizada no Nordeste do Brasil. Assim, os resultados demonstram que a incidência de candidemia foi mais alta que aquela relatada em hospitais públicos brasileiros, e que, como em uma grande série brasileira, Candida albicans, Candida parapsilosis e Candida tropicalis respondem pela maioria dos casos de candidemia. Assim, uma vigilância contínua é necessária para se avaliar as tendências nesta instituição, e estudos mais abrangentes em hospitais privados são necessários para melhor caracterizar a epidemiologia de candidemia nestas unidades de saúde.

\section{REFERÊNCIAS}

1. Alexander JW, Boyce ST, Babcock GF. The process of microbial translocation. Annals of Survey 212: 496-510, 1990.

2. Almirante B, Rodrigues D, Park BJ. Epidemiology and predictor of mortality in cases of Candida bloodstream infection: results from population-based surveillance, Barcelona, Spain, from 2002 to 2003. Journal of Clinical Microbiology 43: 1829-1835, 2005.

3. Antunes AGV, Pasqualotto AC, Diaz MC, Azevedo PA, Severo LC. Candidemia in a Brazilian Tertiary Care Hospital: Species, Distribution and Antifungal Susceptibility Patterns. Revista do Instituto de Medicina Tropical de São Paulo 46: 239-241, 2004.

4. Aquino VR, Lunardi WL, Goldmani LZ, Barth AL. Prevalence, Susceptibility Profile for Fluconazol and Risk Factors for Candidemia in a Tertiary Hospital in Southern Brazil. The Brazilian Journal of Infectious Diseases 9: 411-418, 2005.

5. Barberino MG, Silva N, Rebouças C, Barreiro K, Alcântara AP, Netto EM, Albuquerque L, Brites C. Evaluation of blood stream infections by Candida in three tertiary hospitals in Salvador, Brazil: a case-control study. Brazilian Journal of Infectious Diseases 10: 36-40, 2006.

6. Brito LR, Guimarães T, Nucci M, Rosas RC, Almeida LP, Matta DA, Colombo AL. Clinical and microbiological aspects of candidemia due to C. parapsilosis in Brazilian tertiary care hospitals. Medical Mycology 44: 261-266, 2006.

7. Colombo AL. Epidemiology and treatment of hematogenous candidiasis: a Brazilian prospective. Brazilian Journal Infectious Diseases 4: 113-118, 2000.

8. Colombo AL, Guimarães T. Epidemiology of Hematogenous Infections due to Candida spp. Revista da Sociedade Brasileira de Medicina Tropical 36: 599-607, 2003. 
9. Colombo AL, Nucci M, Park BJ, Nouer AS, Arthington-Skaggs B, Matta DA, Warnock D, Morgan J, Brazilian Network Candidemia Study. Epidemiology of candidemia in Brazil: a nationwide sentinel surveillance of candidemia in eleven medical centers. Journal of Clinical Microbiology 44: 2816-2823, 2006.

10. Edmond MB, Wallace SE, McClish DK, Phaller MA, Jones RN, Wenzel RP. Nosocomial Bloodstream Infections in United State Hospitals: A Three-Year Analysis. Clinical Infectious Diseases 29: 239-244, 1999.

11. França JCB, Ribeiro CEL, Queiroz-Telles F. Candidemia em um hospital terciário brasileiro: incidência, freqüência das diferentes espécies, fatores de risco e suscetibilidade aos antifúngicos. Revista da Sociedade Brasileira de Medicina Tropical 41: 23-28, 2008.

12. Kuse ER, Chetchotisakd P, Cunha CA, Ruhnke M, Barrios C, Raghunadharao D, Sekhon JS, Freire A, Ramasubramanian V, Demeyer I, Nucci M, Leelarasamee A, Jacobs F, Decruvenaere J, Pittet D, Ullmann AJ, Ostrosky-Zeichner L, Lortholary O, Koblinger S, Diekmann-Berndt H, Cornely OA. Micafungin versus liposomal amphotericin B for candidaemia and invasive candidosis: a phase III randomised double-blind trial. Lancet 369: 1519-1527, 2007.

13. Morgan J. Global trends in candidemia: review of reports from 1995-2005. Clinical Infectious Disease Report 7: 429-439, 2005.

14. Nucci M, Colombo AL. Risk Factors for Breakthrough Candidemia. European Journal Microbiology Infectious Diseases 27: 209-211, 2002.

15. Nucci M, Silveira MI, Spector N, Silveira F, Velasco E, Akiti T, Barreiros G, Derossi A, Colombo AL, Pulcheri W. Risk Factors for Death Among Cancer Patients with Fungemia. Clinical Infectious Diseases 27: 107-111, 1998

16. Pappas PG, Rex JH, Lee J, Hamill RJ, Larsen RA, Powderly W, Kauffman CA, Hyslop $\mathrm{N}$, Mangino JE, Chapman S, Horowitz HW, Edwards JE, Dismukes WE. For the NIAID Mycoses Study Group. A Prospective Observational Study of Candidemia.
Epidemiology, Therapy, and Influences on Mortality in Hospitalized Adult and Pediatric Patients. Clinical Infectious Diseases 37: 634-643, 2003.

17. Pasqualotto AC, Nedel WL, Machado TS, Severo LC. A 9-year Study Comparing Risk Factors and the outcome of Pediatrics and Adults with Nosocomial Candidemia. Mycopathologia 160: 111-116, 2005.

18. Pittet D, Li N, Woolson RF, Wenzel RP. Microbiological Factors Influencing of Nosocomial Bloodstream Infections: A 6-Year Validated, Population-Based Model Clinical Infectious Diseases 24: 1068-1078, 1997.

19. Rex JH, Sobel JD. Prophylactic antifungal therapy in the intensive care unit. Clinical Infectious Diseases 32: 1191-1200, 2001.

20. Viudes A, Peman J, Canton E, Úbeda P, Lopez-Ribot JL, Gobernado M. Candidemia at a Terciary-Care Hospital: Epidemiology, Treatment, Clinical Outcome and Risk Factors for Death. European Journal of Microbiology Infectious Diseases 21: 767-774, 2002.

21. Wey SB, Mori M, Pfaller MA, Woolson RF, Wenzel RP. Hospital acquired candidemia. The attributable mortality and excess length of stay. Archives of Internal Medicine 148: 2642-2645, 1998

22. Wingard JR. Importance of Candida species other than Candida albicans as pathogens in oncology patients. Clinical Infectious Diseases 20: 115-125, 1995

23. Wisplinghoff H, Bischoff T, Tallent SM, Seifert H, Wenzel RP, Edmond MB. Nosocomial bloodstream infections in US hospitals: analysis of 24.179 cases from a prospective nationwide surveillance study. Clinical Infectious Diseases 39:309-317, 2004

24. Zeichnner LO, Pappas PG. Invasive candidiases in the intensive care unit. Journal of Critical Care Medicine 34: 857-863, 2006. 